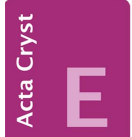
COMMUNICATIONS

ISSN 2056-9890

\section{Crystal and molecular structure of aflatrem}

\author{
Bruno N. Lenta, ${ }^{a}{ }^{*}$ Jules Ngatchou, ${ }^{b}$ Patrice T. Kenfack, ${ }^{c}$ \\ Beate Neumann, ${ }^{d}$ Hans-Georg Stammler ${ }^{d}$ and Norbert \\ Sewald ${ }^{\mathrm{d}}$
}

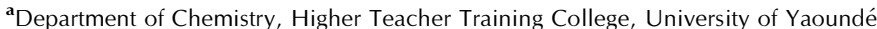
1, PO Box 47, Yaoundé, Cameroon, 'b Department of Organic Chemistry, University of Yaoundé 1, PO Box 812, Yaoundé, Cameroon, ${ }^{\mathbf{c}}$ Department of Inorganic Chemistry, University of Yaoundé 1, PO Box 812, Yaoundé, Cameroon, and dDepartment of Chemistry, University of Bielefeld, PO Box 100131, 33501 Bielefeld, Germany. ${ }^{*}$ Correspondence e-mail: lentabruno@yahoo.fr

Received 13 September 2015; accepted 9 October 2015

Edited by A. J. Lough, University of Toronto, Canada

The crystal structure of the title compound, $\mathrm{C}_{32} \mathrm{H}_{39} \mathrm{NO}_{4}$, confirms the absolute configuration of the seven chiral centres in the molecule. The molecule has a 1,1-dimethylprop-2-enyl substituent on the indole nucleus and this nucleus shares one edge with the five-membered ring which is, in turn, connected to a sequence of three edge-shared fused rings. The skeleton is completed by the 7,7-trimethyl-6,8-dioxabicyclo[3.2.1]oct-3en-2-one group connected to the terminal cyclohexene ring. The two cyclohexane rings adopt chair and half-chair conformations, while in the dioxabicyclo[3.2.1]oct-3-en-2-one unit, the six-membered ring has a half-chair conformation. The indole system of the molecule exhibits a tilt of $2.02(1)^{\circ}$ between its two rings. In the crystal, $\mathrm{O}-\mathrm{H} \cdots \mathrm{O}$ hydrogen bonds connect molecules into chains along [010]. Weak $\mathrm{N}-$ $\mathrm{H} \cdots \pi$ interactions connect these chains, forming sheets parallel to $(10 \overline{1})$.

Keywords: crystal structure; aflatrem; indole-diterpenoid; fungal endophytes; Aspergillus species; $\mathrm{N}-\mathrm{H} \cdots \mathrm{Cg}$ (indole); hydrogen bonding.

CCDC reference: 1430332

\section{Related literature}

For background to indole diterpenoids from endophytes, see: Strobel \& Daisy (2003); Munday-Finch et al. (1996); Gallagher et al. (1980a,b); Lenta et al. (2007); Phongpaichit et al. (2007). For studies of Aspergillus sp, see: Nicholson et al. (2009); Duran et al. (2006). For the pharmacological basis of the behavioural effects of this molecule, see: Tinao-Wooldridge et al. (1995). For the isolation of fungal endophytes from the stem of Symphonia globulifera, see: Petrini et al. (1992); Amin et al. (2014). For geometric details of indole compounds, see: Krishna et al. (1999). For circular dichroism experiments on the title compound, see: Sun et al. (2014). For information on the Cambridge Structural Database (CSD), see: Groom \& Allen (2014).

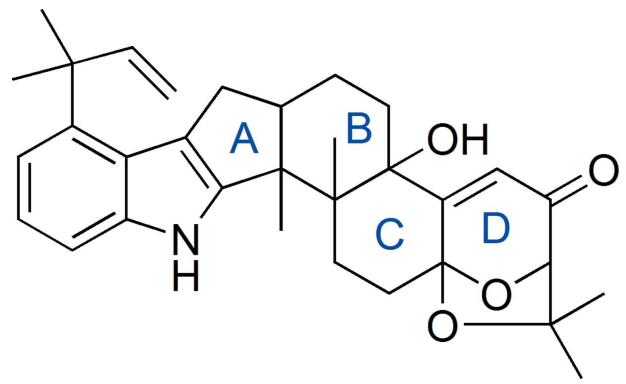

\section{Experimental}

\subsection{Crystal data}

\section{$\mathrm{C}_{32} \mathrm{H}_{39} \mathrm{NO}_{4}$}

$M_{r}=501.64$

Monoclinic, $P 2_{1}$

$a=12.8022(5) \AA$

$b=6.4019$ (2) ^

$c=15.9557(6) \AA$

$\beta=98.821$ (4)

\subsection{Data collection}

Agilent SuperNova Dual Source diffractometer with an Atlas detector

Absorption correction: gaussian (CrysAlis PRO; Agilent, 2013) $T_{\min }=0.899, T_{\max }=1.000$

\subsection{Refinement}

$R\left[F^{2}>2 \sigma\left(F^{2}\right)\right]=0.056$

$w R\left(F^{2}\right)=0.150$

$S=1.06$

4585 reflections

341 parameters

1 restraint

$\mathrm{H}$-atom parameters constrained

$\Delta \rho_{\max }=0.35 \mathrm{e}^{-3}$
$V=1292.24(9) \AA^{3}$

$Z=2$

$\mathrm{Cu} K \alpha$ radiation

$\mu=0.66 \mathrm{~mm}^{-1}$

$T=100 \mathrm{~K}$

$0.18 \times 0.14 \times 0.02 \mathrm{~mm}$
Table 1

Hydrogen-bond geometry $\left(\AA{ }^{\circ}\right)$.

$\mathrm{Cg}$ is the centroid of the $\mathrm{C} 17-\mathrm{C} 22$ ring.

\begin{tabular}{lllll}
\hline$D-\mathrm{H} \cdots A$ & $D-\mathrm{H}$ & $\mathrm{H} \cdots A$ & $D \cdots A$ & $D-\mathrm{H} \cdots A$ \\
\hline $\mathrm{O} 4-\mathrm{H} 4 \cdots \mathrm{O}^{\text {i }}$ & 0.82 & 2.03 & $2.757(3)$ & 148 \\
$\mathrm{~N} 1-\mathrm{H} 1 \cdots C g^{\text {ii }}$ & 0.86 & 2.78 & $3.527(1)$ & 146 \\
\hline
\end{tabular}

Symmetry codes: (i) $-x+2, y-\frac{1}{2},-z+2$; (ii) $-x+1, y-\frac{1}{2},-z+1$.

Data collection: CrysAlis PRO (Agilent, 2013); cell refinement: CrysAlis PRO; data reduction: CrysAlis PRO; program(s) used to solve structure: SHELXS97 (Sheldrick, 2008); program(s) used to refine structure: SHELXL97 (Sheldrick, 2008); molecular graphics: 
DIAMOND (Brandenburg, 1999); software used to prepare material for publication: OLEX2 (Dolomanov et al., 2009).

\section{Acknowledgements}

The authors wish to acknowledge the Alexander von Humboldt Foundation for providing a fellowship to B. N. Lenta at Bielefeld University

Supporting information for this paper is available from the IUCr electronic archives (Reference: LH5789).

\section{References}

Agilent (2013). CrysAlis PRO Agilent Technologies Ltd, Yarnton, Oxfordshire, England.

Amin, N., Salam, M., Junaid, M., Asman \& Baco, M. S. I. (2014). Int J Curr Microbiol Appl Sci. 3, 459-467.

Brandenburg, K. (1999). DIAMOND. Crystal Impact GbR, Bonn, Germany.

Dolomanov, O. V., Bourhis, L. J., Gildea, R. J., Howard, J. A. K. \& Puschmann, H. (2009). J. Appl. Cryst. 42, 339-341.
Duran, R. M., Cary, J. W. \& Calvo, A. M. (2006). Appl. Microbiol. Biotechnol. 73, $1158-1168$

Gallagher, R. T., Clardy, J. \& Wilson, B. J. (1980b). Tetrahedron Lett. 21, 239242.

Gallagher, R. T., Finer, J., Clardy, J., Leutwiler, A., Weibel, F., Acklin, W. \& Arigoni, D. (1980a). Tetrahedron Lett. 21, 235-238.

Groom, C. R. \& Allen, F. H. (2014). Angew. Chem. Int. Ed. 53, 662-671.

Krishna, R., Velmurugan, D., Babu, G. \& Perumal, P. T. (1999). Acta Cryst. C55, 75-78.

Lenta, B. N., Noungoue, D. T., Devkota, K. P., Fokou, P. A., Ngouela, S., Tsamo, E. \& Sewald, N. (2007). Acta Cryst. E63, o1282-o1284.

Munday-Finch, S. C., Wilkins, A. L. \& Miles, C. O. (1996). Phytochemistry, 41, 327-332.

Nicholson, M. J., Koulman, A., Monahan, B. J., Pritchard, B. L., Payne, G. A. \& Scott, B. (2009). Appl. Environ. Microbiol. 75, 7469-7481.

Parsons, S. \& Flack, H. D. (2004). Acta Cryst. A60, s61.

Petrini, O., Sueber, I., Titi, L. \& Viret, O. (1992). Nat. Toxins, 1, 185-196.

Phongpaichit, S., Nikom, J., Rungjindamai, N., Sakayaroj, J., HutadilokTowatana, N., Rukachaisirikul, V. \& Kirtikara, K. (2007). FEMS Immunol. Med. Microbiol. 51, 517-525.

Sheldrick, G. M. (2008). Acta Cryst. A64, 112-122.

Strobel, G. \& Daisy, B. (2003). Microbiol. Mol. Biol. Rev. 67, 491-502.

Sun, K., Li, Y., Guo, L., Wang, Y., Liu, P. \& Zhu, W. (2014). Mar. Drugs, 12, 3970-3981.

Tinao-Wooldridge, L. V., Hsiang, B. C. H., Latifi, T. N., Ferrendelli, J. A. \& Covey, D. F. (1995). Bioorg. Med. Chem. Lett. 5, 265-270. 


\section{supporting information}

Acta Cryst. (2015). E71, o867-o868 [https://doi.org/10.1107/S2056989015019040]

\section{Crystal and molecular structure of aflatrem}

\section{Bruno N. Lenta, Jules Ngatchou, Patrice T. Kenfack, Beate Neumann, Hans-Georg Stammler and Norbert Sewald}

\section{S1. Comment}

The search of compounds from plant endophytes has been the subject of research interest (Strobel \& Daisy 2003). They produce a plethora of substances with potential applications in agriculture, medicine, pharmaceutical and for industry (Petrini et al. 1992; Strobel \& Daisy, 2003; Phongpaichit et al., 2007). We are interested in the isolation and the structural study of compounds produced by endophytes from Cameroonian medicinal plants with pharmacological properties (Lenta et al., 2007) and one of the compounds that we have isolated from the fungal endophyte of the stem of Symphonia globulifera was aflatrem. Some authors have identified the biosynthetic genes of this molecule in Aspergilius sp (Nicholson et al., 2009; Duran et al., 2006). We report herein the study of its molecular and crystal structure.

Aflatrem crystallizes in the non-centrosymmetric space group $P 2_{1}$ and its asymmetric unit consists of a single molecule as shown in Figure 1. As known in the literature, the molecule has a 1,1-dimethyl-2-propenyl substituent on the indole nucleus at position $\mathrm{C} 18$ and this nucleus shares one edge with the 5-membered ring (A) belonging to a group of three fused rings like an anthracene system (see Fig.1). The two others rings (6-membered, B and C) also share one edge with the 7, 7-trimethyl-6,8- dioxabicyclo[3.2.1]oct-3-en-2-one group. All the bond distances observed in the compounds are in agreement with the bonds distance of the Cambridge Structural Database (CSD, Groom \& Allen, 2014). In the indole ring system, a small tilt of $2.02(1)^{\circ}$ is observed between the 6 and 5 -membered rings. This value as well as the values of the bond angles is near the values always obtained in the indole based compounds (Krishna et al., 1999). The ring A $(\mathrm{C} 1 / \mathrm{C} 16 / \mathrm{C} 15 / \mathrm{C} 14 / \mathrm{C} 2)$ adopts a dihedral angle of $3.12(1)^{\circ}$ with the 5-membered ring of indole system. With the exception of the $\mathrm{C} 1-\mathrm{C} 2-\mathrm{C} 14$ angle $\left(96.9(1)^{\circ}\right)$,the values of the bond angles in this ring are in the range between 100 and $112^{\circ}$ and they are in good agreement with the ideal conformation for which the angle is $107^{\circ}$. The lower value observed could be favored by the chair conformation of the $\mathrm{B}$ ring which shares one edge ( $\mathrm{C} 2$ and $\mathrm{C} 14)$ with ring $\mathrm{A}$. This conformation is close to the ideal chair conformation since the bond angles range from 107 to $113^{\circ}$ compared to an ideal value of $109^{\circ}$. The ring $\mathrm{C}$ assumes a half-chair conformation. The bicyclo[3.2.1]oct-3-en-2-one system is composed by a 6-membered ring named $\mathrm{D}(\mathrm{C} 6 / \mathrm{C} 10 / \mathrm{C} 9 / \mathrm{C} 8 / \mathrm{C} 7 / \mathrm{O} 1)$ sharing one edge $(\mathrm{C} 6-\mathrm{C} 7)$ with a 5-membered ring called $\mathrm{E}$ $(\mathrm{O} 1 / \mathrm{C} 7 / \mathrm{C} 6 / \mathrm{O} 2 / \mathrm{C} 25)$. The carbon atoms of ring $\mathrm{D}$ lie in the same plane and the $\mathrm{O} 1$ atom is located at 0.80 (1) $\AA$ from this plane. This atom is also located at 0.656 (1) $\AA$ from the plane which contains the carbon atoms of ring E and the dihedral angle between the two planes is $69.07(1)^{\circ}$. The methyl and hydroxyl groups linked to the fused ring give the absolute configuration of $1 \mathrm{~S}, 3 R, 6 \mathrm{~S}, 7 S, 11 R, 12 S, 13 \mathrm{~S}$ determined by $\mathrm{Cu} \mathrm{K} \alpha X$-radiation with the Flack parameter being refined to 0.09 (14) and this configuration is in agreement with the previous circular dichroism assignment reported by Sun et al. (2014).

The crystal packing of the aflatrem molecules is illustrated in Figs. 2 and 3. In the crystal, molecules are connected along the $b$ axis via $\mathrm{O}-\mathrm{H} \cdots \mathrm{O}$ hydrogen bonds. In addition, weak $\mathrm{N}-\mathrm{H} \cdots \pi$ (indole) interactions connect these chains 
forming planes parallel to (10-1). This $\mathrm{N}-\mathrm{H} \cdots \pi$ (indole) interaction is typical of indole-based molecules as reported by Krishna et al. (1999).

\section{S2. Experimental}

The isolation of fungal endophytes from the stem of Symphonia globulifera was carried out aat the University of Yaoundé 1 (Cameroon) and was based on the method described by Petrini et al. (1992). One of the fungi was identified to Aspergillus sp. according to the method described by Amin et al. (2014) and cultured in solid medium prepared from $1 \mathrm{~kg}$ of rice distributed in the glass flask (total capacity of $2.5 \mathrm{~L}$ ) at a rate of $200 \mathrm{~g}$ of rice in $200 \mathrm{ml}$ of distilled water. After one month of incubation at $301 \mathrm{~K}$ in the same laboratory, the culture medium was extracted with EtOAc and the extract concentrated on a rotary evaporator under vacuum at a temperature of $313 \mathrm{~K}$ to yield $20.1 \mathrm{~g}$ of extract. This extract was subjected to column chromatography (CC) over silica gel (0.023-0.20 mesh, Merck) and eluted with a gradient system of petroleum ether /ethyl acetate to afford aflatrem $(7.5 \mathrm{mg})$. The colourless crystals obtained were sent to the Laboratory of Inorganic and Structural Chemistry at Bielefeld University (Germany) for X-ray diffraction measurements.

\section{S3. Refinement}

$\mathrm{H}$ atoms were placed in calculated positions with $\mathrm{C}-\mathrm{H}=0.93-0.98 \AA, \mathrm{N}-\mathrm{H}=0.86 \AA$ and $\mathrm{O}-\mathrm{H}=0.82 \AA$. They were included in calculated positions with $\mathrm{U}_{\text {iso }}(\mathrm{H})=1.2 \mathrm{U}_{\text {eq }}(\mathrm{C})$ or $1.5 \mathrm{U}_{\text {eq }}\left(\mathrm{C}_{\text {methyl }}, \mathrm{O}\right)$.

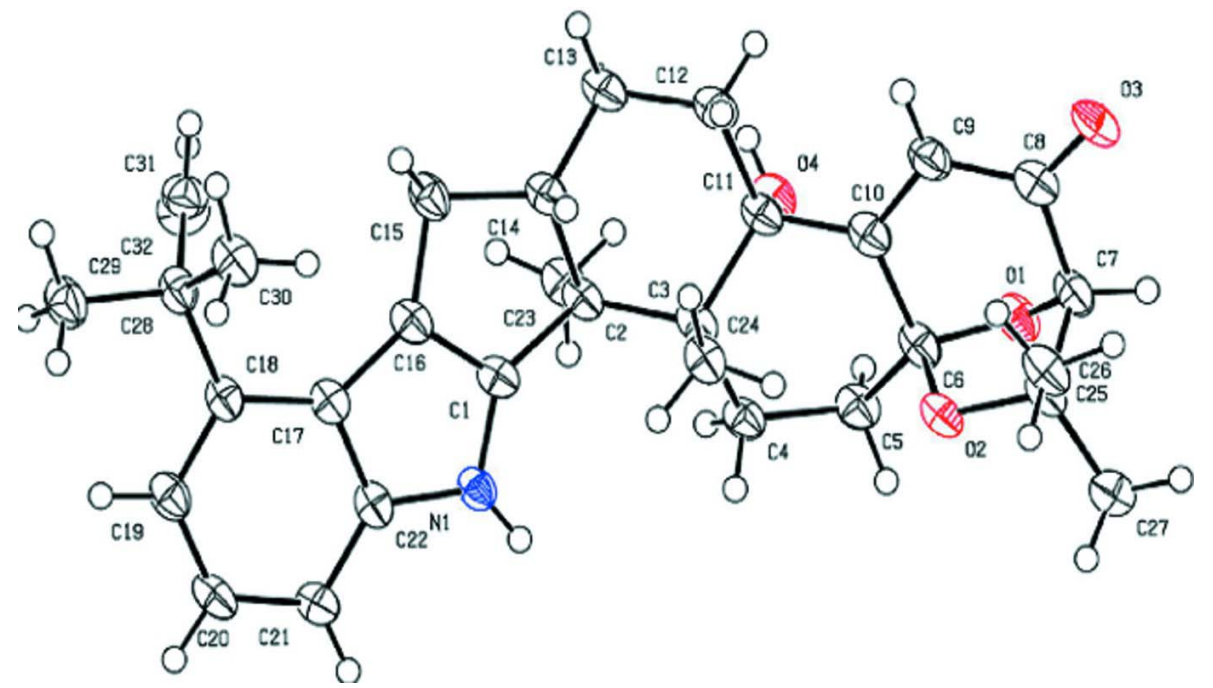

Figure 1

The molecular structure of aflatrem with the atom-labelling scheme. Displacement ellipsoids are drawn at the 50\% probability level. 


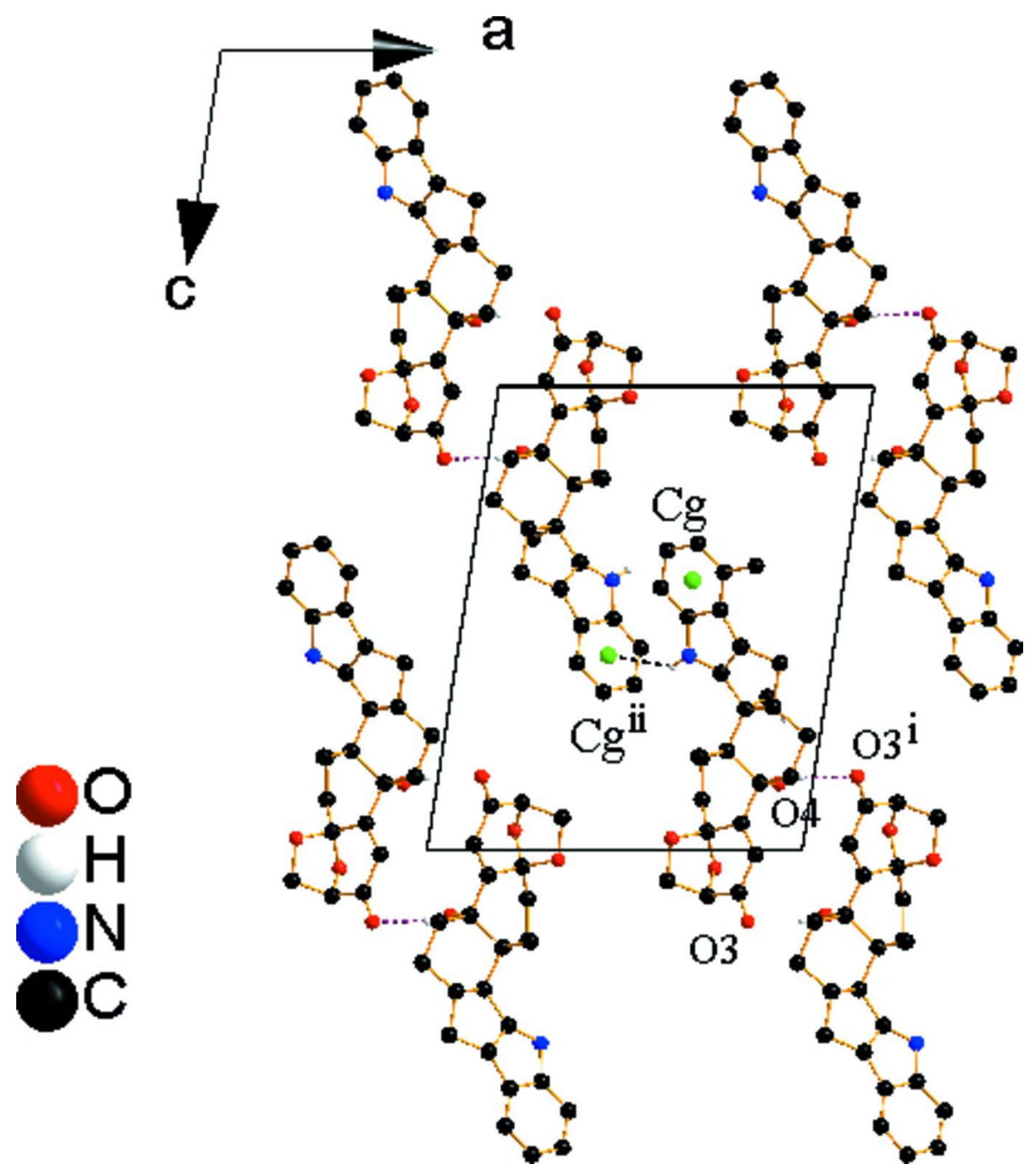

Figure 2

Crystal packing of aflatrem showing $\mathrm{O}-\mathrm{H} \cdots \mathrm{O}$ hydrogen-bonded (dashed lines) zigzag chains along the $b$ axis in the (010) plane. Weak $\mathrm{N}-\mathrm{H} \cdots \pi$ interactions are also shown as dashed lines. Symmetry codes: (i) $-x+2, y-1 / 2,-z+2$; (ii) $-x$ $+1, y-1 / 2,-z+1$; (iii) $-x+1, y+1 / 2,-z+1$; (iv) $-x+2, y+1 / 2,-z+2$. 


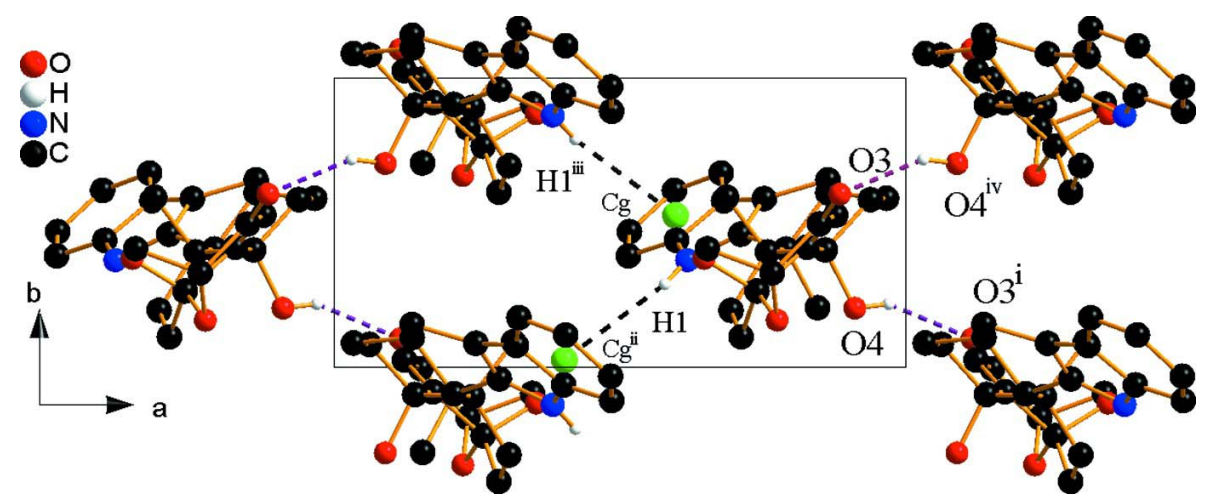

Figure 3

Crystal packing of aflatrem showing $\mathrm{O}-\mathrm{H} \cdots \mathrm{O}$ hydrogen-bonded (dashed lines) zigzag chains along the $b$ axis in the (001) plane. Weak $\mathrm{N}-\mathrm{H} \cdots \pi$ interactions are also shown as dashed lines. Symmetry codes: (i) $-x+2, y-1 / 2,-z+2$; (ii) $-x$ $+1, y-1 / 2,-z+1$; (iii) $-x+1, y+1 / 2,-z+1$ and (iv) $-x+2, y+1 / 2,-z+2$.

(3R,5bS,7aS,13bS,13cR,15aS)-9-(1,1-Dimethyl-2-propenyl)-2,3,5b,6,7,7a,8,13,13b,13c,14,15-dodecahydro-5bhydroxy-2,2,13b,13c-tetramethyl-4H-3,15a-epoxy-1-benzoxepino $\left[6^{\prime}, 7^{\prime}: 6,7\right]$ indeno[1,2-b] indol-4-one

Crystal data

$\mathrm{C}_{32} \mathrm{H}_{39} \mathrm{NO}_{4}$

$M_{r}=501.64$

Monoclinic, $P 2_{1}$

$a=12.8022(5) \AA$

$b=6.4019(2) \AA$

$c=15.9557(6) \AA$

$\beta=98.821(4)^{\circ}$

$V=1292.24(9) \AA^{3}$

$Z=2$

\section{Data collection}

Agilent SuperNova Dual Source diffractometer with an Atlas detector

Radiation source: SuperNova $(\mathrm{Cu}) \mathrm{X}$-ray Source

Mirror monochromator

Detector resolution: 5.3114 pixels $\mathrm{mm}^{-1}$

$\omega$ scans

Absorption correction: gaussian

(CrysAlis PRO; Agilent, 2013)

Refinement

Refinement on $F^{2}$

Least-squares matrix: full

$R\left[F^{2}>2 \sigma\left(F^{2}\right)\right]=0.056$

$w R\left(F^{2}\right)=0.150$

$S=1.06$

4585 reflections

341 parameters

1 restraint

Primary atom site location: structure-invariant direct methods
$F(000)=540$

$D_{\mathrm{x}}=1.289 \mathrm{Mg} \mathrm{m}^{-3}$

$\mathrm{Cu} K \alpha$ radiation, $\lambda=1.5418 \AA$

Cell parameters from 6775 reflections

$\theta=4.8-66.4^{\circ}$

$\mu=0.66 \mathrm{~mm}^{-1}$

$T=100 \mathrm{~K}$

Plate, colourless

$0.18 \times 0.14 \times 0.02 \mathrm{~mm}$

$T_{\min }=0.899, T_{\max }=1.000$

19981 measured reflections

4585 independent reflections

4078 reflections with $I>2 \sigma(I)$

$R_{\text {int }}=0.050$

$\theta_{\max }=66.9^{\circ}, \theta_{\min }=2.8^{\circ}$

$h=-15 \rightarrow 15$

$k=-7 \rightarrow 7$

$l=-18 \rightarrow 18$

Hydrogen site location: inferred from neighbouring sites

$\mathrm{H}$-atom parameters constrained

$w=1 /\left[\sigma^{2}\left(F_{\mathrm{o}}^{2}\right)+(0.0948 P)^{2}+0.4383 P\right]$

where $P=\left(F_{\mathrm{o}}{ }^{2}+2 F_{\mathrm{c}}{ }^{2}\right) / 3$

$(\Delta / \sigma)_{\max }<0.001$

$\Delta \rho_{\max }=0.35$ e $\AA^{-3}$

$\Delta \rho_{\min }=-0.23$ e $\AA^{-3}$ 
Absolute structure: Flack $x$ determined using

1671 quotients $\left[\left(I^{+}\right)-(I)\right] /\left[\left(I^{+}\right)+(I)\right]$ (Parsons \&

Flack, 2004)

Absolute structure parameter: 0.09 (14)

Special details

Experimental. Numerical absorption correction based on gaussian integration over a multifaceted crystal model Geometry. All e.s.d.'s (except the e.s.d. in the dihedral angle between two 1.s. planes) are estimated using the full covariance matrix. The cell e.s.d.'s are taken into account individually in the estimation of e.s.d.'s in distances, angles and torsion angles; correlations between e.s.d.'s in cell parameters are only used when they are defined by crystal symmetry. An approximate (isotropic) treatment of cell e.s.d.'s is used for estimating e.s.d.'s involving 1.s. planes.

Fractional atomic coordinates and isotropic or equivalent isotropic displacement parameters $\left(\AA^{2}\right)$

\begin{tabular}{|c|c|c|c|c|}
\hline & $x$ & $y$ & $z$ & $U_{\text {iso }} * / U_{\text {eq }}$ \\
\hline $\mathrm{O} 1$ & $0.77480(17)$ & $0.1640(3)$ & $1.04104(13)$ & $0.0330(5)$ \\
\hline $\mathrm{O} 2$ & $0.64687(16)$ & $0.3772(3)$ & $0.97693(13)$ & $0.0307(5)$ \\
\hline $\mathrm{O} 3$ & $0.88440(18)$ & $0.5994(4)$ & 1.15707 (14) & $0.0414(6)$ \\
\hline $\mathrm{O} 4$ & $0.91050(17)$ & $0.2056(3)$ & $0.85758(13)$ & $0.0316(5)$ \\
\hline $\mathrm{H} 4$ & 0.9698 & 0.2203 & 0.8449 & $0.047 *$ \\
\hline N1 & 0.61755 (19) & $0.3716(4)$ & $0.58123(15)$ & $0.0267(5)$ \\
\hline H1 & 0.5782 & 0.2885 & 0.6050 & $0.032 *$ \\
\hline $\mathrm{C} 1$ & $0.7110(2)$ & $0.4610(4)$ & 0.61906 (19) & $0.0260(6)$ \\
\hline $\mathrm{C} 2$ & $0.7906(2)$ & $0.4099(4)$ & 0.69719 (18) & $0.0252(6)$ \\
\hline $\mathrm{C} 3$ & $0.7579(2)$ & $0.4000(5)$ & $0.78767(18)$ & $0.0264(6)$ \\
\hline $\mathrm{C} 4$ & $0.6942(2)$ & $0.1981(4)$ & 0.80235 (19) & $0.0279(6)$ \\
\hline $\mathrm{H} 4 \mathrm{~A}$ & 0.6195 & 0.2288 & 0.7870 & $0.033 *$ \\
\hline H4B & 0.7117 & 0.0912 & 0.7637 & $0.033^{*}$ \\
\hline $\mathrm{C} 5$ & $0.7119(2)$ & $0.1065(5)$ & 0.89279 (19) & $0.0301(6)$ \\
\hline $\mathrm{H} 5 \mathrm{~A}$ & 0.6482 & 0.0338 & 0.9021 & $0.036^{*}$ \\
\hline $\mathrm{H} 5 \mathrm{~B}$ & 0.7684 & 0.0044 & 0.8968 & $0.036^{*}$ \\
\hline C6 & $0.7394(2)$ & $0.2648(5)$ & $0.96202(19)$ & $0.0293(7)$ \\
\hline $\mathrm{C} 7$ & $0.7658(3)$ & $0.3273(5)$ & 1.10039 (19) & $0.0327(7)$ \\
\hline H7 & 0.7714 & 0.2729 & 1.1583 & $0.039 *$ \\
\hline $\mathrm{C} 8$ & $0.8486(2)$ & $0.4967(5)$ & $1.0940(2)$ & $0.0338(7)$ \\
\hline C9 & $0.8771(2)$ & $0.5268(5)$ & $1.0107(2)$ & $0.0328(7)$ \\
\hline H9 & 0.9297 & 0.6218 & 1.0028 & $0.039 *$ \\
\hline $\mathrm{C} 10$ & $0.8270(2)$ & $0.4165(5)$ & $0.94420(19)$ & $0.0287(6)$ \\
\hline $\mathrm{C} 11$ & $0.8592(2)$ & $0.4071(5)$ & $0.85721(18)$ & $0.0270(6)$ \\
\hline $\mathrm{C} 12$ & $0.9380(2)$ & $0.5804(5)$ & $0.8432(2)$ & $0.0316(7)$ \\
\hline $\mathrm{H} 12 \mathrm{~A}$ & 0.9080 & 0.7144 & 0.8551 & $0.038 *$ \\
\hline H12B & 1.0022 & 0.5614 & 0.8834 & $0.038^{*}$ \\
\hline C13 & $0.9661(2)$ & $0.5851(5)$ & $0.75408(19)$ & $0.0326(7)$ \\
\hline H13A & 1.0117 & 0.7032 & 0.7477 & $0.039 *$ \\
\hline H13B & 1.0029 & 0.4580 & 0.7430 & $0.039 *$ \\
\hline C14 & $0.8634(2)$ & $0.6041(5)$ & $0.69266(19)$ & $0.0277(6)$ \\
\hline H14 & 0.8256 & 0.7216 & 0.7135 & $0.033 *$ \\
\hline $\mathrm{C} 15$ & $0.8636(2)$ & $0.6488(5)$ & $0.59791(19)$ & $0.0313(7)$ \\
\hline H15A & 0.8759 & 0.7955 & 0.5876 & $0.038^{*}$ \\
\hline
\end{tabular}




\begin{tabular}{|c|c|c|c|c|}
\hline H15B & 0.9157 & 0.5649 & 0.5751 & $0.038^{*}$ \\
\hline $\mathrm{C} 16$ & $0.7522(2)$ & $0.5841(4)$ & $0.56234(19)$ & $0.0276(6)$ \\
\hline $\mathrm{C} 17$ & $0.6812(2)$ & $0.5766(4)$ & $0.48313(18)$ & $0.0262(6)$ \\
\hline $\mathrm{C} 18$ & $0.6770(2)$ & $0.6725(4)$ & $0.40181(18)$ & $0.0267(6)$ \\
\hline $\mathrm{C} 19$ & $0.5951(2)$ & $0.6126(5)$ & $0.33970(19)$ & $0.0310(7)$ \\
\hline H19 & 0.5918 & 0.6687 & 0.2856 & $0.037 *$ \\
\hline $\mathrm{C} 20$ & $0.5165(2)$ & $0.4704(5)$ & $0.35503(19)$ & $0.0308(7)$ \\
\hline $\mathrm{H} 20$ & 0.4644 & 0.4318 & 0.3105 & $0.037 *$ \\
\hline $\mathrm{C} 21$ & $0.5147(2)$ & $0.3863(5)$ & $0.43453(19)$ & $0.0279(6)$ \\
\hline $\mathrm{H} 21$ & 0.4605 & 0.2986 & 0.4455 & $0.033 *$ \\
\hline $\mathrm{C} 22$ & $0.5987(2)$ & $0.4402(4)$ & $0.49762(18)$ & $0.0262(6)$ \\
\hline $\mathrm{C} 23$ & $0.8453(3)$ & $0.2072(5)$ & $0.6718(2)$ & $0.0308(7)$ \\
\hline $\mathrm{H} 23 \mathrm{~A}$ & 0.7937 & 0.0983 & 0.6598 & $0.046^{*}$ \\
\hline $\mathrm{H} 23 \mathrm{~B}$ & 0.8987 & 0.1648 & 0.7176 & $0.046^{*}$ \\
\hline $\mathrm{H} 23 \mathrm{C}$ & 0.8774 & 0.2338 & 0.6223 & $0.046^{*}$ \\
\hline $\mathrm{C} 24$ & $0.6862(2)$ & 0.5888 (4) & $0.80049(19)$ & $0.0281(6)$ \\
\hline $\mathrm{H} 24 \mathrm{~A}$ & 0.7270 & 0.7150 & 0.8035 & $0.042 *$ \\
\hline H24B & 0.6576 & 0.5712 & 0.8523 & $0.042 *$ \\
\hline $\mathrm{H} 24 \mathrm{C}$ & 0.6294 & 0.5971 & 0.7537 & $0.042 *$ \\
\hline $\mathrm{C} 25$ & $0.6530(2)$ & $0.4108(6)$ & $1.06813(19)$ & $0.0336(7)$ \\
\hline $\mathrm{C} 26$ & $0.6353(3)$ & $0.6379(5)$ & $1.0847(2)$ & $0.0384(8)$ \\
\hline H26A & 0.6817 & 0.7211 & 1.0565 & $0.058^{*}$ \\
\hline H26B & 0.6497 & 0.6639 & 1.1447 & $0.058^{*}$ \\
\hline $\mathrm{H} 26 \mathrm{C}$ & 0.5633 & 0.6738 & 1.0637 & $0.058^{*}$ \\
\hline $\mathrm{C} 27$ & $0.5722(3)$ & $0.2731(6)$ & $1.1015(2)$ & $0.0376(8)$ \\
\hline H27A & 0.5028 & 0.3075 & 1.0731 & $0.056^{*}$ \\
\hline H27B & 0.5757 & 0.2954 & 1.1614 & $0.056^{*}$ \\
\hline $\mathrm{H} 27 \mathrm{C}$ & 0.5872 & 0.1292 & 1.0912 & $0.056^{*}$ \\
\hline $\mathrm{C} 28$ & $0.7593(2)$ & $0.8372(4)$ & $0.38782(18)$ & $0.0295(7)$ \\
\hline C29 & $0.7411(3)$ & $0.9297(5)$ & $0.2981(2)$ & $0.0381(8)$ \\
\hline $\mathrm{H} 29 \mathrm{~A}$ & 0.7483 & 0.8217 & 0.2576 & $0.057^{*}$ \\
\hline $\mathrm{H} 29 \mathrm{~B}$ & 0.7924 & 1.0372 & 0.2940 & $0.057^{*}$ \\
\hline $\mathrm{H} 29 \mathrm{C}$ & 0.6714 & 0.9881 & 0.2866 & $0.057^{*}$ \\
\hline C30 & $0.7512(3)$ & $1.0194(5)$ & $0.4492(2)$ & $0.0351(7)$ \\
\hline $\mathrm{H} 30 \mathrm{~A}$ & 0.6870 & 1.0954 & 0.4313 & $0.053^{*}$ \\
\hline H30B & 0.8106 & 1.1109 & 0.4494 & $0.053^{*}$ \\
\hline $\mathrm{H} 30 \mathrm{C}$ & 0.7508 & 0.9657 & 0.5054 & $0.053^{*}$ \\
\hline C31 & $0.8695(3)$ & $0.7438(5)$ & $0.4004(2)$ & 0.0340 \\
\hline H31 & 0.9255 & 0.8328 & 0.4195 & $0.041 *$ \\
\hline $\mathrm{C} 32$ & 0.8925 (3) & $0.5482(6)$ & $0.3866(2)$ & $0.0426(8)$ \\
\hline $\mathrm{H} 32 \mathrm{~A}$ & 0.8388 & 0.4540 & 0.3675 & $0.051^{*}$ \\
\hline H32B & 0.9625 & 0.5039 & 0.3960 & $0.051 *$ \\
\hline
\end{tabular}

Atomic displacement parameters $\left(\AA^{2}\right)$

\begin{tabular}{lllllll}
\hline & $U^{11}$ & $U^{22}$ & $U^{33}$ & $U^{12}$ & $U^{13}$ & $U^{23}$ \\
\hline O1 & $0.0397(12)$ & $0.0260(10)$ & $0.0297(11)$ & $0.0012(9)$ & $-0.0062(9)$ & $0.0028(9)$ \\
O2 & $0.0311(10)$ & $0.0307(11)$ & $0.0269(10)$ & $-0.0007(9)$ & $-0.0065(8)$ & $-0.0031(9)$
\end{tabular}




\begin{tabular}{|c|c|c|c|c|c|c|}
\hline $\mathrm{O} 3$ & $0.0390(12)$ & 0.0489 (14) & $0.0317(12)$ & $-0.0085(11)$ & $-0.0090(10)$ & $-0.0092(11)$ \\
\hline O4 & $0.0329(11)$ & $0.0272(11)$ & $0.0327(11)$ & $0.0059(9)$ & $-0.0019(9)$ & $0.0019(9)$ \\
\hline N1 & $0.0308(12)$ & $0.0218(12)$ & $0.0254(12)$ & $-0.0015(10)$ & $-0.0027(10)$ & $0.0024(10)$ \\
\hline $\mathrm{C} 1$ & $0.0273(14)$ & $0.0188(13)$ & $0.0298(15)$ & $0.0013(11)$ & $-0.0018(12)$ & $-0.0022(11)$ \\
\hline $\mathrm{C} 2$ & $0.0296(14)$ & $0.0163(12)$ & $0.0270(14)$ & $0.0012(12)$ & $-0.0037(11)$ & $-0.0015(11)$ \\
\hline C3 & 0.0304 (14) & $0.0177(13)$ & $0.0276(15)$ & $0.0005(12)$ & $-0.0062(12)$ & $-0.0004(11)$ \\
\hline $\mathrm{C} 4$ & $0.0310(15)$ & $0.0198(14)$ & $0.0303(14)$ & $-0.0030(12)$ & $-0.0031(12)$ & $-0.0014(11)$ \\
\hline $\mathrm{C} 5$ & $0.0368(16)$ & $0.0186(13)$ & $0.0330(16)$ & $-0.0041(12)$ & $-0.0006(13)$ & $-0.0036(12)$ \\
\hline C6 & $0.0351(16)$ & $0.0229(15)$ & $0.0268(15)$ & $0.0005(12)$ & $-0.0055(12)$ & $-0.0014(11)$ \\
\hline C7 & $0.0348(16)$ & $0.0349(16)$ & $0.0250(15)$ & $-0.0017(13)$ & $-0.0065(12)$ & $0.0008(13)$ \\
\hline $\mathrm{C} 8$ & $0.0299(15)$ & $0.0364(17)$ & $0.0325(16)$ & $0.0013(13)$ & $-0.0034(13)$ & $-0.0014(13)$ \\
\hline $\mathrm{C} 9$ & $0.0330(15)$ & $0.0330(16)$ & $0.0296(15)$ & $-0.0062(13)$ & $-0.0044(12)$ & $-0.0008(13)$ \\
\hline $\mathrm{C} 10$ & 0.0307 (14) & $0.0212(14)$ & $0.0314(15)$ & $0.0026(12)$ & $-0.0038(12)$ & $0.0015(12)$ \\
\hline C11 & $0.0281(14)$ & $0.0216(13)$ & $0.0286(15)$ & $0.0006(12)$ & $-0.0044(12)$ & $-0.0014(12)$ \\
\hline C12 & $0.0316(15)$ & $0.0278(15)$ & $0.0325(16)$ & $-0.0030(13)$ & $-0.0047(12)$ & $-0.0014(13)$ \\
\hline C13 & $0.0306(15)$ & $0.0311(15)$ & $0.0330(16)$ & $-0.0027(13)$ & $-0.0055(13)$ & $-0.0035(13)$ \\
\hline $\mathrm{C} 14$ & $0.0285(14)$ & $0.0231(14)$ & $0.0290(15)$ & $-0.0015(12)$ & $-0.0037(12)$ & $0.0008(12)$ \\
\hline C15 & $0.0386(17)$ & $0.0234(14)$ & $0.0283(15)$ & $-0.0022(12)$ & $-0.0056(13)$ & $0.0026(12)$ \\
\hline $\mathrm{C} 16$ & $0.0322(14)$ & $0.0193(13)$ & $0.0292(15)$ & $0.0002(12)$ & $-0.0021(12)$ & $-0.0022(12)$ \\
\hline C17 & $0.0307(14)$ & $0.0206(13)$ & $0.0260(14)$ & 0.0018 (12) & $-0.0001(12)$ & $-0.0008(11)$ \\
\hline $\mathrm{C} 18$ & $0.0329(15)$ & $0.0207(13)$ & $0.0247(14)$ & $-0.0001(12)$ & $-0.0007(11)$ & $0.0007(11)$ \\
\hline C19 & $0.0353(15)$ & $0.0265(15)$ & $0.0287(15)$ & $0.0036(13)$ & $-0.0034(13)$ & $0.0026(12)$ \\
\hline $\mathrm{C} 20$ & $0.0329(15)$ & $0.0247(14)$ & $0.0300(15)$ & $0.0019(12)$ & $-0.0099(13)$ & $-0.0009(12)$ \\
\hline $\mathrm{C} 21$ & $0.0293(14)$ & $0.0200(13)$ & $0.0318(15)$ & $-0.0002(12)$ & $-0.0032(12)$ & $0.0019(12)$ \\
\hline $\mathrm{C} 22$ & $0.0327(15)$ & $0.0190(14)$ & $0.0254(14)$ & $0.0028(11)$ & $-0.0006(12)$ & $-0.0004(11)$ \\
\hline $\mathrm{C} 23$ & $0.0365(16)$ & $0.0212(15)$ & $0.0326(15)$ & $0.0024(12)$ & $-0.0016(13)$ & $-0.0028(12)$ \\
\hline $\mathrm{C} 24$ & $0.0362(16)$ & $0.0183(13)$ & $0.0280(14)$ & $0.0027(12)$ & $-0.0013(12)$ & $-0.0020(11)$ \\
\hline $\mathrm{C} 25$ & $0.0339(16)$ & $0.0382(17)$ & $0.0255(15)$ & $-0.0009(14)$ & $-0.0059(12)$ & $-0.0034(13)$ \\
\hline $\mathrm{C} 26$ & $0.0408(17)$ & $0.0387(18)$ & $0.0317(16)$ & $0.0011(14)$ & $-0.0074(13)$ & $-0.0077(14)$ \\
\hline $\mathrm{C} 27$ & $0.0336(17)$ & $0.0419(19)$ & $0.0354(17)$ & $-0.0041(14)$ & $-0.0010(14)$ & $-0.0025(14)$ \\
\hline $\mathrm{C} 28$ & $0.0384(16)$ & $0.0222(15)$ & $0.0262(15)$ & $-0.0027(12)$ & $-0.0007(12)$ & $0.0031(12)$ \\
\hline $\mathrm{C} 29$ & $0.0478(19)$ & $0.0319(17)$ & $0.0310(16)$ & $-0.0070(14)$ & $-0.0054(14)$ & $0.0056(13)$ \\
\hline C30 & $0.0453(18)$ & $0.0211(15)$ & $0.0359(17)$ & $-0.0015(13)$ & $-0.0034(14)$ & $0.0009(13)$ \\
\hline C31 & $0.0332(16)$ & $0.0319(16)$ & $0.0349(16)$ & $-0.0061(13)$ & $-0.0013(13)$ & $0.0006(13)$ \\
\hline $\mathrm{C} 32$ & $0.0397(18)$ & $0.0356(18)$ & $0.052(2)$ & $0.0042(15)$ & $0.0068(16)$ & $0.0025(15)$ \\
\hline
\end{tabular}

Geometric parameters $\left(\AA,{ }^{\circ}\right)$

\begin{tabular}{llll}
\hline $\mathrm{O} 1-\mathrm{C} 7$ & $1.427(4)$ & $\mathrm{C} 15-\mathrm{C} 16$ & $1.509(4)$ \\
$\mathrm{O} 1-\mathrm{C} 6$ & $1.427(4)$ & $\mathrm{C} 15-\mathrm{H} 15 \mathrm{~A}$ & 0.9700 \\
$\mathrm{O} 2-\mathrm{C} 6$ & $1.437(4)$ & $\mathrm{C} 15-\mathrm{H} 15 \mathrm{~B}$ & 0.9700 \\
$\mathrm{O} 2-\mathrm{C} 25$ & $1.461(4)$ & $\mathrm{C} 16-\mathrm{C} 17$ & $1.440(4)$ \\
$\mathrm{O} 3-\mathrm{C} 8$ & $1.231(4)$ & $\mathrm{C} 17-\mathrm{C} 22$ & $1.417(4)$ \\
$\mathrm{O} 4-\mathrm{C} 11$ & $1.447(4)$ & $\mathrm{C} 17-\mathrm{C} 18$ & $1.429(4)$ \\
$\mathrm{O} 4-\mathrm{H} 4$ & 0.8200 & $\mathrm{C} 18-\mathrm{C} 19$ & $1.382(4)$ \\
$\mathrm{N} 1-\mathrm{C} 1$ & $1.379(4)$ & $\mathrm{C} 18-\mathrm{C} 28$ & $1.532(4)$ \\
$\mathrm{N} 1-\mathrm{C} 22$ & $1.390(4)$ & $\mathrm{C} 19-\mathrm{C} 20$ & $1.406(5)$ \\
$\mathrm{N} 1-\mathrm{H} 1$ & 0.8600 & $\mathrm{C} 19-\mathrm{H} 19$ & 0.9300
\end{tabular}




\begin{tabular}{|c|c|c|c|}
\hline $\mathrm{C} 1-\mathrm{C} 16$ & $1.366(4)$ & $\mathrm{C} 20-\mathrm{C} 21$ & $1.381(4)$ \\
\hline $\mathrm{C} 1-\mathrm{C} 2$ & $1.520(4)$ & $\mathrm{C} 20-\mathrm{H} 20$ & 0.9300 \\
\hline $\mathrm{C} 2-\mathrm{C} 23$ & $1.557(4)$ & $\mathrm{C} 21-\mathrm{C} 22$ & $1.399(4)$ \\
\hline $\mathrm{C} 2-\mathrm{C} 14$ & $1.562(4)$ & $\mathrm{C} 21-\mathrm{H} 21$ & 0.9300 \\
\hline $\mathrm{C} 2-\mathrm{C} 3$ & $1.565(4)$ & $\mathrm{C} 23-\mathrm{H} 23 \mathrm{~A}$ & 0.9600 \\
\hline $\mathrm{C} 3-\mathrm{C} 24$ & $1.550(4)$ & $\mathrm{C} 23-\mathrm{H} 23 \mathrm{~B}$ & 0.9600 \\
\hline $\mathrm{C} 3-\mathrm{C} 4$ & $1.565(4)$ & $\mathrm{C} 23-\mathrm{H} 23 \mathrm{C}$ & 0.9600 \\
\hline $\mathrm{C} 3-\mathrm{C} 11$ & $1.573(4)$ & $\mathrm{C} 24-\mathrm{H} 24 \mathrm{~A}$ & 0.9600 \\
\hline $\mathrm{C} 4-\mathrm{C} 5$ & $1.542(4)$ & $\mathrm{C} 24-\mathrm{H} 24 \mathrm{~B}$ & 0.9600 \\
\hline $\mathrm{C} 4-\mathrm{H} 4 \mathrm{~A}$ & 0.9700 & $\mathrm{C} 24-\mathrm{H} 24 \mathrm{C}$ & 0.9600 \\
\hline $\mathrm{C} 4-\mathrm{H} 4 \mathrm{~B}$ & 0.9700 & $\mathrm{C} 25-\mathrm{C} 26$ & $1.501(5)$ \\
\hline $\mathrm{C} 5-\mathrm{C} 6$ & $1.500(4)$ & $\mathrm{C} 25-\mathrm{C} 27$ & $1.517(5)$ \\
\hline $\mathrm{C} 5-\mathrm{H} 5 \mathrm{~A}$ & 0.9700 & $\mathrm{C} 26-\mathrm{H} 26 \mathrm{~A}$ & 0.9600 \\
\hline C5-H5B & 0.9700 & $\mathrm{C} 26-\mathrm{H} 26 \mathrm{~B}$ & 0.9600 \\
\hline $\mathrm{C} 6-\mathrm{C} 10$ & $1.543(4)$ & $\mathrm{C} 26-\mathrm{H} 26 \mathrm{C}$ & 0.9600 \\
\hline $\mathrm{C} 7-\mathrm{C} 8$ & $1.531(5)$ & C27-H27A & 0.9600 \\
\hline $\mathrm{C} 7-\mathrm{C} 25$ & $1.552(4)$ & $\mathrm{C} 27-\mathrm{H} 27 \mathrm{~B}$ & 0.9600 \\
\hline $\mathrm{C} 7-\mathrm{H} 7$ & 0.9800 & $\mathrm{C} 27-\mathrm{H} 27 \mathrm{C}$ & 0.9600 \\
\hline $\mathrm{C} 8-\mathrm{C} 9$ & $1.443(5)$ & $\mathrm{C} 28-\mathrm{C} 31$ & $1.516(4)$ \\
\hline $\mathrm{C} 9-\mathrm{C} 10$ & $1.351(4)$ & $\mathrm{C} 28-\mathrm{C} 29$ & $1.533(4)$ \\
\hline $\mathrm{C} 9-\mathrm{H} 9$ & 0.9300 & $\mathrm{C} 28-\mathrm{C} 30$ & $1.537(4)$ \\
\hline $\mathrm{C} 10-\mathrm{C} 11$ & $1.508(4)$ & $\mathrm{C} 29-\mathrm{H} 29 \mathrm{~A}$ & 0.9600 \\
\hline $\mathrm{C} 11-\mathrm{C} 12$ & $1.538(4)$ & $\mathrm{C} 29-\mathrm{H} 29 \mathrm{~B}$ & 0.9600 \\
\hline $\mathrm{C} 12-\mathrm{C} 13$ & $1.521(4)$ & $\mathrm{C} 29-\mathrm{H} 29 \mathrm{C}$ & 0.9600 \\
\hline $\mathrm{C} 12-\mathrm{H} 12 \mathrm{~A}$ & 0.9700 & $\mathrm{C} 30-\mathrm{H} 30 \mathrm{~A}$ & 0.9600 \\
\hline $\mathrm{C} 12-\mathrm{H} 12 \mathrm{~B}$ & 0.9700 & $\mathrm{C} 30-\mathrm{H} 30 \mathrm{~B}$ & 0.9600 \\
\hline $\mathrm{C} 13-\mathrm{C} 14$ & $1.520(4)$ & $\mathrm{C} 30-\mathrm{H} 30 \mathrm{C}$ & 0.9600 \\
\hline C13-H13A & 0.9700 & C31-C32 & $1.313(5)$ \\
\hline C13-H13B & 0.9700 & $\mathrm{C} 31-\mathrm{H} 31$ & 0.9300 \\
\hline $\mathrm{C} 14-\mathrm{C} 15$ & $1.539(4)$ & $\mathrm{C} 32-\mathrm{H} 32 \mathrm{~A}$ & 0.9300 \\
\hline $\mathrm{C} 14-\mathrm{H} 14$ & 0.9800 & $\mathrm{C} 32-\mathrm{H} 32 \mathrm{~B}$ & 0.9300 \\
\hline $\mathrm{C} 7-\mathrm{O} 1-\mathrm{C} 6$ & $102.0(2)$ & $\mathrm{C} 14-\mathrm{C} 15-\mathrm{H} 15 \mathrm{~A}$ & 111.8 \\
\hline $\mathrm{C} 6-\mathrm{O} 2-\mathrm{C} 25$ & $108.6(2)$ & $\mathrm{C} 16-\mathrm{C} 15-\mathrm{H} 15 \mathrm{~B}$ & 111.8 \\
\hline $\mathrm{C} 11-\mathrm{O} 4-\mathrm{H} 4$ & 109.5 & C14-C15-H15B & 111.8 \\
\hline $\mathrm{C} 1-\mathrm{N} 1-\mathrm{C} 22$ & $107.5(2)$ & $\mathrm{H} 15 \mathrm{~A}-\mathrm{C} 15-\mathrm{H} 15 \mathrm{~B}$ & 109.5 \\
\hline $\mathrm{C} 1-\mathrm{N} 1-\mathrm{H} 1$ & 126.3 & $\mathrm{C} 1-\mathrm{C} 16-\mathrm{C} 17$ & $107.9(3)$ \\
\hline $\mathrm{C} 22-\mathrm{N} 1-\mathrm{H} 1$ & 126.3 & $\mathrm{C} 1-\mathrm{C} 16-\mathrm{C} 15$ & $110.4(3)$ \\
\hline $\mathrm{C} 16-\mathrm{C} 1-\mathrm{N} 1$ & $110.3(2)$ & $\mathrm{C} 17-\mathrm{C} 16-\mathrm{C} 15$ & $140.5(3)$ \\
\hline $\mathrm{C} 16-\mathrm{C} 1-\mathrm{C} 2$ & $112.9(2)$ & $\mathrm{C} 22-\mathrm{C} 17-\mathrm{C} 18$ & $119.1(3)$ \\
\hline $\mathrm{N} 1-\mathrm{C} 1-\mathrm{C} 2$ & $134.2(3)$ & $\mathrm{C} 22-\mathrm{C} 17-\mathrm{C} 16$ & $105.3(2)$ \\
\hline $\mathrm{C} 1-\mathrm{C} 2-\mathrm{C} 23$ & $103.7(2)$ & $\mathrm{C} 18-\mathrm{C} 17-\mathrm{C} 16$ & $135.6(3)$ \\
\hline $\mathrm{C} 1-\mathrm{C} 2-\mathrm{C} 14$ & $96.9(2)$ & $\mathrm{C} 19-\mathrm{C} 18-\mathrm{C} 17$ & $116.8(3)$ \\
\hline $\mathrm{C} 23-\mathrm{C} 2-\mathrm{C} 14$ & $110.8(2)$ & $\mathrm{C} 19-\mathrm{C} 18-\mathrm{C} 28$ & $123.4(3)$ \\
\hline $\mathrm{C} 1-\mathrm{C} 2-\mathrm{C} 3$ & $121.6(2)$ & $\mathrm{C} 17-\mathrm{C} 18-\mathrm{C} 28$ & $119.8(2)$ \\
\hline $\mathrm{C} 23-\mathrm{C} 2-\mathrm{C} 3$ & $113.7(2)$ & $\mathrm{C} 18-\mathrm{C} 19-\mathrm{C} 20$ & $122.6(3)$ \\
\hline $\mathrm{C} 14-\mathrm{C} 2-\mathrm{C} 3$ & $108.7(2)$ & $\mathrm{C} 18-\mathrm{C} 19-\mathrm{H} 19$ & 118.7 \\
\hline $\mathrm{C} 24-\mathrm{C} 3-\mathrm{C} 2$ & $109.7(2)$ & $\mathrm{C} 20-\mathrm{C} 19-\mathrm{H} 19$ & 118.7 \\
\hline
\end{tabular}




\begin{tabular}{|c|c|}
\hline $\mathrm{C} 24-\mathrm{C} 3-\mathrm{C} 4$ & $106.9(2)$ \\
\hline $\mathrm{C} 2-\mathrm{C} 3-\mathrm{C} 4$ & $113.0(2)$ \\
\hline $\mathrm{C} 24-\mathrm{C} 3-\mathrm{C} 11$ & $109.0(2)$ \\
\hline $\mathrm{C} 2-\mathrm{C} 3-\mathrm{C} 11$ & $109.9(2)$ \\
\hline $\mathrm{C} 4-\mathrm{C} 3-\mathrm{C} 11$ & $108.1(2)$ \\
\hline $\mathrm{C} 5-\mathrm{C} 4-\mathrm{C} 3$ & $116.7(2)$ \\
\hline $\mathrm{C} 5-\mathrm{C} 4-\mathrm{H} 4 \mathrm{~A}$ & 108.1 \\
\hline $\mathrm{C} 3-\mathrm{C} 4-\mathrm{H} 4 \mathrm{~A}$ & 108.1 \\
\hline $\mathrm{C} 5-\mathrm{C} 4-\mathrm{H} 4 \mathrm{~B}$ & 108.1 \\
\hline $\mathrm{C} 3-\mathrm{C} 4-\mathrm{H} 4 \mathrm{~B}$ & 108.1 \\
\hline $\mathrm{H} 4 \mathrm{~A}-\mathrm{C} 4-\mathrm{H} 4 \mathrm{~B}$ & 107.3 \\
\hline $\mathrm{C} 6-\mathrm{C} 5-\mathrm{C} 4$ & $114.6(2)$ \\
\hline $\mathrm{C} 6-\mathrm{C} 5-\mathrm{H} 5 \mathrm{~A}$ & 108.6 \\
\hline $\mathrm{C} 4-\mathrm{C} 5-\mathrm{H} 5 \mathrm{~A}$ & 108.6 \\
\hline $\mathrm{C} 6-\mathrm{C} 5-\mathrm{H} 5 \mathrm{~B}$ & 108.6 \\
\hline $\mathrm{C} 4-\mathrm{C} 5-\mathrm{H} 5 \mathrm{~B}$ & 108.6 \\
\hline $\mathrm{H} 5 \mathrm{~A}-\mathrm{C} 5-\mathrm{H} 5 \mathrm{~B}$ & 107.6 \\
\hline $\mathrm{O} 1-\mathrm{C} 6-\mathrm{O} 2$ & $103.8(2)$ \\
\hline $\mathrm{O} 1-\mathrm{C} 6-\mathrm{C} 5$ & $110.6(2)$ \\
\hline $\mathrm{O} 2-\mathrm{C} 6-\mathrm{C} 5$ & $110.7(2)$ \\
\hline $\mathrm{O} 1-\mathrm{C} 6-\mathrm{C} 10$ & $107.8(2)$ \\
\hline $\mathrm{O} 2-\mathrm{C} 6-\mathrm{C} 10$ & $110.9(2)$ \\
\hline $\mathrm{C} 5-\mathrm{C} 6-\mathrm{C} 10$ & $112.6(3)$ \\
\hline $\mathrm{O} 1-\mathrm{C} 7-\mathrm{C} 8$ & $110.3(3)$ \\
\hline $\mathrm{O} 1-\mathrm{C} 7-\mathrm{C} 25$ & $101.4(2)$ \\
\hline $\mathrm{C} 8-\mathrm{C} 7-\mathrm{C} 25$ & $110.6(3)$ \\
\hline $\mathrm{O} 1-\mathrm{C} 7-\mathrm{H} 7$ & 111.4 \\
\hline $\mathrm{C} 8-\mathrm{C} 7-\mathrm{H} 7$ & 111.4 \\
\hline $\mathrm{C} 25-\mathrm{C} 7-\mathrm{H} 7$ & 111.4 \\
\hline $\mathrm{O} 3-\mathrm{C} 8-\mathrm{C} 9$ & $124.6(3)$ \\
\hline $\mathrm{O} 3-\mathrm{C} 8-\mathrm{C} 7$ & $119.9(3)$ \\
\hline $\mathrm{C} 9-\mathrm{C} 8-\mathrm{C} 7$ & $115.5(3)$ \\
\hline $\mathrm{C} 10-\mathrm{C} 9-\mathrm{C} 8$ & $119.8(3)$ \\
\hline $\mathrm{C} 10-\mathrm{C} 9-\mathrm{H} 9$ & 120.1 \\
\hline $\mathrm{C} 8-\mathrm{C} 9-\mathrm{H} 9$ & 120.1 \\
\hline $\mathrm{C} 9-\mathrm{C} 10-\mathrm{C} 11$ & $125.4(3)$ \\
\hline $\mathrm{C} 9-\mathrm{C} 10-\mathrm{C} 6$ & $117.1(3)$ \\
\hline $\mathrm{C} 11-\mathrm{C} 10-\mathrm{C} 6$ & $116.9(3)$ \\
\hline $\mathrm{O} 4-\mathrm{C} 11-\mathrm{C} 10$ & $102.7(2)$ \\
\hline $\mathrm{O} 4-\mathrm{C} 11-\mathrm{C} 12$ & $109.6(2)$ \\
\hline $\mathrm{C} 10-\mathrm{C} 11-\mathrm{C} 12$ & $112.5(2)$ \\
\hline $\mathrm{O} 4-\mathrm{C} 11-\mathrm{C} 3$ & $107.6(2)$ \\
\hline $\mathrm{C} 10-\mathrm{C} 11-\mathrm{C} 3$ & $109.8(2)$ \\
\hline $\mathrm{C} 12-\mathrm{C} 11-\mathrm{C} 3$ & $114.0(2)$ \\
\hline $\mathrm{C} 13-\mathrm{C} 12-\mathrm{C} 11$ & $113.9(2)$ \\
\hline $\mathrm{C} 13-\mathrm{C} 12-\mathrm{H} 12 \mathrm{~A}$ & 108.8 \\
\hline $\mathrm{C} 11-\mathrm{C} 12-\mathrm{H} 12 \mathrm{~A}$ & 108.8 \\
\hline $\mathrm{C} 13-\mathrm{C} 12-\mathrm{H} 12 \mathrm{~B}$ & 1088 \\
\hline
\end{tabular}

\begin{tabular}{|c|c|}
\hline $\mathrm{C} 21-\mathrm{C} 20-\mathrm{C} 19$ & $121.7(3)$ \\
\hline $\mathrm{C} 21-\mathrm{C} 20-\mathrm{H} 20$ & 119.2 \\
\hline $\mathrm{C} 19-\mathrm{C} 20-\mathrm{H} 20$ & 119.2 \\
\hline $\mathrm{C} 20-\mathrm{C} 21-\mathrm{C} 22$ & $116.5(3)$ \\
\hline $\mathrm{C} 20-\mathrm{C} 21-\mathrm{H} 21$ & 121.8 \\
\hline $\mathrm{C} 22-\mathrm{C} 21-\mathrm{H} 21$ & 121.8 \\
\hline $\mathrm{N} 1-\mathrm{C} 22-\mathrm{C} 21$ & $127.9(3)$ \\
\hline $\mathrm{N} 1-\mathrm{C} 22-\mathrm{C} 17$ & $109.1(2)$ \\
\hline $\mathrm{C} 21-\mathrm{C} 22-\mathrm{C} 17$ & $123.0(3)$ \\
\hline $\mathrm{C} 2-\mathrm{C} 23-\mathrm{H} 23 \mathrm{~A}$ & 109.5 \\
\hline $\mathrm{C} 2-\mathrm{C} 23-\mathrm{H} 23 \mathrm{~B}$ & 109.5 \\
\hline $\mathrm{H} 23 \mathrm{~A}-\mathrm{C} 23-\mathrm{H} 23 \mathrm{~B}$ & 109.5 \\
\hline $\mathrm{C} 2-\mathrm{C} 23-\mathrm{H} 23 \mathrm{C}$ & 109.5 \\
\hline $\mathrm{H} 23 \mathrm{~A}-\mathrm{C} 23-\mathrm{H} 23 \mathrm{C}$ & 109.5 \\
\hline $\mathrm{H} 23 \mathrm{~B}-\mathrm{C} 23-\mathrm{H} 23 \mathrm{C}$ & 109.5 \\
\hline $\mathrm{C} 3-\mathrm{C} 24-\mathrm{H} 24 \mathrm{~A}$ & 109.5 \\
\hline $\mathrm{C} 3-\mathrm{C} 24-\mathrm{H} 24 \mathrm{~B}$ & 109.5 \\
\hline $\mathrm{H} 24 \mathrm{~A}-\mathrm{C} 24-\mathrm{H} 24 \mathrm{~B}$ & 109.5 \\
\hline $\mathrm{C} 3-\mathrm{C} 24-\mathrm{H} 24 \mathrm{C}$ & 109.5 \\
\hline $\mathrm{H} 24 \mathrm{~A}-\mathrm{C} 24-\mathrm{H} 24 \mathrm{C}$ & 109.5 \\
\hline $\mathrm{H} 24 \mathrm{~B}-\mathrm{C} 24-\mathrm{H} 24 \mathrm{C}$ & 109.5 \\
\hline $\mathrm{O} 2-\mathrm{C} 25-\mathrm{C} 26$ & $109.4(3)$ \\
\hline $\mathrm{O} 2-\mathrm{C} 25-\mathrm{C} 27$ & $109.2(3)$ \\
\hline $\mathrm{C} 26-\mathrm{C} 25-\mathrm{C} 27$ & $111.8(3)$ \\
\hline $\mathrm{O} 2-\mathrm{C} 25-\mathrm{C} 7$ & $100.7(2)$ \\
\hline $\mathrm{C} 26-\mathrm{C} 25-\mathrm{C} 7$ & $115.7(3)$ \\
\hline $\mathrm{C} 27-\mathrm{C} 25-\mathrm{C} 7$ & $109.5(3)$ \\
\hline $\mathrm{C} 25-\mathrm{C} 26-\mathrm{H} 26 \mathrm{~A}$ & 109.5 \\
\hline $\mathrm{C} 25-\mathrm{C} 26-\mathrm{H} 26 \mathrm{~B}$ & 109.5 \\
\hline $\mathrm{H} 26 \mathrm{~A}-\mathrm{C} 26-\mathrm{H} 26 \mathrm{~B}$ & 109.5 \\
\hline $\mathrm{C} 25-\mathrm{C} 26-\mathrm{H} 26 \mathrm{C}$ & 109.5 \\
\hline $\mathrm{H} 26 \mathrm{~A}-\mathrm{C} 26-\mathrm{H} 26 \mathrm{C}$ & 109.5 \\
\hline $\mathrm{H} 26 \mathrm{~B}-\mathrm{C} 26-\mathrm{H} 26 \mathrm{C}$ & 109.5 \\
\hline $\mathrm{C} 25-\mathrm{C} 27-\mathrm{H} 27 \mathrm{~A}$ & 109.5 \\
\hline $\mathrm{C} 25-\mathrm{C} 27-\mathrm{H} 27 \mathrm{~B}$ & 109.5 \\
\hline $\mathrm{H} 27 \mathrm{~A}-\mathrm{C} 27-\mathrm{H} 27 \mathrm{~B}$ & 109.5 \\
\hline $\mathrm{C} 25-\mathrm{C} 27-\mathrm{H} 27 \mathrm{C}$ & 109.5 \\
\hline $\mathrm{H} 27 \mathrm{~A}-\mathrm{C} 27-\mathrm{H} 27 \mathrm{C}$ & 109.5 \\
\hline $\mathrm{H} 27 \mathrm{~B}-\mathrm{C} 27-\mathrm{H} 27 \mathrm{C}$ & 109.5 \\
\hline $\mathrm{C} 31-\mathrm{C} 28-\mathrm{C} 18$ & $110.9(2)$ \\
\hline $\mathrm{C} 31-\mathrm{C} 28-\mathrm{C} 29$ & $106.3(3)$ \\
\hline $\mathrm{C} 18-\mathrm{C} 28-\mathrm{C} 29$ & $113.1(3)$ \\
\hline $\mathrm{C} 31-\mathrm{C} 28-\mathrm{C} 30$ & $111.6(3)$ \\
\hline $\mathrm{C} 18-\mathrm{C} 28-\mathrm{C} 30$ & $108.3(2)$ \\
\hline $\mathrm{C} 29-\mathrm{C} 28-\mathrm{C} 30$ & $106.7(3)$ \\
\hline $\mathrm{C} 28-\mathrm{C} 29-\mathrm{H} 29 \mathrm{~A}$ & 109.5 \\
\hline $\mathrm{C} 28-\mathrm{C} 29-\mathrm{H} 29 \mathrm{~B}$ & 109.5 \\
\hline $\mathrm{H} 29 \mathrm{~A}-\mathrm{C} 29-\mathrm{H} 29 \mathrm{~B}$ & 109.5 \\
\hline
\end{tabular}




\begin{tabular}{|c|c|c|c|}
\hline $\mathrm{C} 11-\mathrm{C} 12-\mathrm{H} 12 \mathrm{~B}$ & 108.8 & $\mathrm{C} 28-\mathrm{C} 29-\mathrm{H} 29 \mathrm{C}$ & 109.5 \\
\hline $\mathrm{H} 12 \mathrm{~A}-\mathrm{C} 12-\mathrm{H} 12 \mathrm{~B}$ & 107.7 & $\mathrm{H} 29 \mathrm{~A}-\mathrm{C} 29-\mathrm{H} 29 \mathrm{C}$ & 109.5 \\
\hline $\mathrm{C} 14-\mathrm{C} 13-\mathrm{C} 12$ & $107.4(2)$ & $\mathrm{H} 29 \mathrm{~B}-\mathrm{C} 29-\mathrm{H} 29 \mathrm{C}$ & 109.5 \\
\hline $\mathrm{C} 14-\mathrm{C} 13-\mathrm{H} 13 \mathrm{~A}$ & 110.2 & $\mathrm{C} 28-\mathrm{C} 30-\mathrm{H} 30 \mathrm{~A}$ & 109.5 \\
\hline $\mathrm{C} 12-\mathrm{C} 13-\mathrm{H} 13 \mathrm{~A}$ & 110.2 & $\mathrm{C} 28-\mathrm{C} 30-\mathrm{H} 30 \mathrm{~B}$ & 109.5 \\
\hline $\mathrm{C} 14-\mathrm{C} 13-\mathrm{H} 13 \mathrm{~B}$ & 110.2 & $\mathrm{H} 30 \mathrm{~A}-\mathrm{C} 30-\mathrm{H} 30 \mathrm{~B}$ & 109.5 \\
\hline $\mathrm{C} 12-\mathrm{C} 13-\mathrm{H} 13 \mathrm{~B}$ & 110.2 & $\mathrm{C} 28-\mathrm{C} 30-\mathrm{H} 30 \mathrm{C}$ & 109.5 \\
\hline $\mathrm{H} 13 \mathrm{~A}-\mathrm{C} 13-\mathrm{H} 13 \mathrm{~B}$ & 108.5 & $\mathrm{H} 30 \mathrm{~A}-\mathrm{C} 30-\mathrm{H} 30 \mathrm{C}$ & 109.5 \\
\hline $\mathrm{C} 13-\mathrm{C} 14-\mathrm{C} 15$ & $121.1(3)$ & $\mathrm{H} 30 \mathrm{~B}-\mathrm{C} 30-\mathrm{H} 30 \mathrm{C}$ & 109.5 \\
\hline $\mathrm{C} 13-\mathrm{C} 14-\mathrm{C} 2$ & $111.7(2)$ & $\mathrm{C} 32-\mathrm{C} 31-\mathrm{C} 28$ & $125.6(3)$ \\
\hline $\mathrm{C} 15-\mathrm{C} 14-\mathrm{C} 2$ & $106.5(2)$ & $\mathrm{C} 32-\mathrm{C} 31-\mathrm{H} 31$ & 117.2 \\
\hline $\mathrm{C} 13-\mathrm{C} 14-\mathrm{H} 14$ & 105.4 & $\mathrm{C} 28-\mathrm{C} 31-\mathrm{H} 31$ & 117.2 \\
\hline $\mathrm{C} 15-\mathrm{C} 14-\mathrm{H} 14$ & 105.4 & $\mathrm{C} 31-\mathrm{C} 32-\mathrm{H} 32 \mathrm{~A}$ & 120.0 \\
\hline $\mathrm{C} 2-\mathrm{C} 14-\mathrm{H} 14$ & 105.4 & $\mathrm{C} 31-\mathrm{C} 32-\mathrm{H} 32 \mathrm{~B}$ & 120.0 \\
\hline $\mathrm{C} 16-\mathrm{C} 15-\mathrm{C} 14$ & $100.1(2)$ & $\mathrm{H} 32 \mathrm{~A}-\mathrm{C} 32-\mathrm{H} 32 \mathrm{~B}$ & 120.0 \\
\hline $\mathrm{C} 16-\mathrm{C} 15-\mathrm{H} 15 \mathrm{~A}$ & 111.8 & & \\
\hline $\mathrm{C} 22-\mathrm{N} 1-\mathrm{C} 1-\mathrm{C} 16$ & $0.0(3)$ & $\mathrm{C} 4-\mathrm{C} 3-\mathrm{C} 11-\mathrm{C} 12$ & $171.7(2)$ \\
\hline $\mathrm{C} 22-\mathrm{N} 1-\mathrm{C} 1-\mathrm{C} 2$ & $-159.8(3)$ & $\mathrm{O} 4-\mathrm{C} 11-\mathrm{C} 12-\mathrm{C} 13$ & $70.5(3)$ \\
\hline $\mathrm{C} 16-\mathrm{C} 1-\mathrm{C} 2-\mathrm{C} 23$ & $-88.8(3)$ & $\mathrm{C} 10-\mathrm{C} 11-\mathrm{C} 12-\mathrm{C} 13$ & $-175.9(2)$ \\
\hline $\mathrm{N} 1-\mathrm{C} 1-\mathrm{C} 2-\mathrm{C} 23$ & $70.7(4)$ & $\mathrm{C} 3-\mathrm{C} 11-\mathrm{C} 12-\mathrm{C} 13$ & $-50.1(3)$ \\
\hline $\mathrm{C} 16-\mathrm{C} 1-\mathrm{C} 2-\mathrm{C} 14$ & $24.7(3)$ & $\mathrm{C} 11-\mathrm{C} 12-\mathrm{C} 13-\mathrm{C} 14$ & $55.7(3)$ \\
\hline $\mathrm{N} 1-\mathrm{C} 1-\mathrm{C} 2-\mathrm{C} 14$ & $-175.9(3)$ & $\mathrm{C} 12-\mathrm{C} 13-\mathrm{C} 14-\mathrm{C} 15$ & $170.0(3)$ \\
\hline $\mathrm{C} 16-\mathrm{C} 1-\mathrm{C} 2-\mathrm{C} 3$ & $141.7(3)$ & $\mathrm{C} 12-\mathrm{C} 13-\mathrm{C} 14-\mathrm{C} 2$ & $-63.3(3)$ \\
\hline $\mathrm{N} 1-\mathrm{C} 1-\mathrm{C} 2-\mathrm{C} 3$ & $-58.8(4)$ & $\mathrm{C} 1-\mathrm{C} 2-\mathrm{C} 14-\mathrm{C} 13$ & $-169.4(2)$ \\
\hline $\mathrm{C} 1-\mathrm{C} 2-\mathrm{C} 3-\mathrm{C} 24$ & $-44.3(3)$ & $\mathrm{C} 23-\mathrm{C} 2-\mathrm{C} 14-\mathrm{C} 13$ & $-61.8(3)$ \\
\hline $\mathrm{C} 23-\mathrm{C} 2-\mathrm{C} 3-\mathrm{C} 24$ & $-169.4(2)$ & $\mathrm{C} 3-\mathrm{C} 2-\mathrm{C} 14-\mathrm{C} 13$ & $63.8(3)$ \\
\hline $\mathrm{C} 14-\mathrm{C} 2-\mathrm{C} 3-\mathrm{C} 24$ & $66.6(3)$ & $\mathrm{C} 1-\mathrm{C} 2-\mathrm{C} 14-\mathrm{C} 15$ & $-35.2(3)$ \\
\hline $\mathrm{C} 1-\mathrm{C} 2-\mathrm{C} 3-\mathrm{C} 4$ & $74.9(3)$ & $\mathrm{C} 23-\mathrm{C} 2-\mathrm{C} 14-\mathrm{C} 15$ & $72.4(3)$ \\
\hline $\mathrm{C} 23-\mathrm{C} 2-\mathrm{C} 3-\mathrm{C} 4$ & $-50.1(3)$ & $\mathrm{C} 3-\mathrm{C} 2-\mathrm{C} 14-\mathrm{C} 15$ & $-162.0(2)$ \\
\hline $\mathrm{C} 14-\mathrm{C} 2-\mathrm{C} 3-\mathrm{C} 4$ & $-174.1(2)$ & $\mathrm{C} 13-\mathrm{C} 14-\mathrm{C} 15-\mathrm{C} 16$ & $162.6(3)$ \\
\hline $\mathrm{C} 1-\mathrm{C} 2-\mathrm{C} 3-\mathrm{C} 11$ & $-164.2(2)$ & $\mathrm{C} 2-\mathrm{C} 14-\mathrm{C} 15-\mathrm{C} 16$ & $33.6(3)$ \\
\hline $\mathrm{C} 23-\mathrm{C} 2-\mathrm{C} 3-\mathrm{C} 11$ & $70.7(3)$ & $\mathrm{N} 1-\mathrm{C} 1-\mathrm{C} 16-\mathrm{C} 17$ & $0.6(3)$ \\
\hline $\mathrm{C} 14-\mathrm{C} 2-\mathrm{C} 3-\mathrm{C} 11$ & $-53.2(3)$ & $\mathrm{C} 2-\mathrm{C} 1-\mathrm{C} 16-\mathrm{C} 17$ & $165.0(2)$ \\
\hline $\mathrm{C} 24-\mathrm{C} 3-\mathrm{C} 4-\mathrm{C} 5$ & $-93.2(3)$ & $\mathrm{N} 1-\mathrm{C} 1-\mathrm{C} 16-\mathrm{C} 15$ & $-169.2(2)$ \\
\hline $\mathrm{C} 2-\mathrm{C} 3-\mathrm{C} 4-\mathrm{C} 5$ & $145.9(3)$ & $\mathrm{C} 2-\mathrm{C} 1-\mathrm{C} 16-\mathrm{C} 15$ & $-4.8(3)$ \\
\hline $\mathrm{C} 11-\mathrm{C} 3-\mathrm{C} 4-\mathrm{C} 5$ & $24.0(3)$ & $\mathrm{C} 14-\mathrm{C} 15-\mathrm{C} 16-\mathrm{C} 1$ & $-18.0(3)$ \\
\hline $\mathrm{C} 3-\mathrm{C} 4-\mathrm{C} 5-\mathrm{C} 6$ & $30.1(4)$ & $\mathrm{C} 14-\mathrm{C} 15-\mathrm{C} 16-\mathrm{C} 17$ & $177.3(4)$ \\
\hline $\mathrm{C} 7-\mathrm{O} 1-\mathrm{C} 6-\mathrm{O} 2$ & $-44.4(3)$ & $\mathrm{C} 1-\mathrm{C} 16-\mathrm{C} 17-\mathrm{C} 22$ & $-0.9(3)$ \\
\hline $\mathrm{C} 7-\mathrm{O} 1-\mathrm{C} 6-\mathrm{C} 5$ & $-163.2(3)$ & $\mathrm{C} 15-\mathrm{C} 16-\mathrm{C} 17-\mathrm{C} 22$ & $164.0(4)$ \\
\hline $\mathrm{C} 7-\mathrm{O} 1-\mathrm{C} 6-\mathrm{C} 10$ & $73.3(3)$ & $\mathrm{C} 1-\mathrm{C} 16-\mathrm{C} 17-\mathrm{C} 18$ & $177.8(3)$ \\
\hline $\mathrm{C} 25-\mathrm{O} 2-\mathrm{C} 6-\mathrm{O} 1$ & $22.5(3)$ & $\mathrm{C} 15-\mathrm{C} 16-\mathrm{C} 17-\mathrm{C} 18$ & $-17.3(6)$ \\
\hline $\mathrm{C} 25-\mathrm{O} 2-\mathrm{C} 6-\mathrm{C} 5$ & $141.2(2)$ & $\mathrm{C} 22-\mathrm{C} 17-\mathrm{C} 18-\mathrm{C} 19$ & $-4.7(4)$ \\
\hline $\mathrm{C} 25-\mathrm{O} 2-\mathrm{C} 6-\mathrm{C} 10$ & $-93.0(3)$ & $\mathrm{C} 16-\mathrm{C} 17-\mathrm{C} 18-\mathrm{C} 19$ & $176.7(3)$ \\
\hline $\mathrm{C} 4-\mathrm{C} 5-\mathrm{C} 6-\mathrm{O} 1$ & $-168.4(2)$ & $\mathrm{C} 22-\mathrm{C} 17-\mathrm{C} 18-\mathrm{C} 28$ & $173.7(3)$ \\
\hline $\mathrm{C} 4-\mathrm{C} 5-\mathrm{C} 6-\mathrm{O} 2$ & $77.1(3)$ & $\mathrm{C} 16-\mathrm{C} 17-\mathrm{C} 18-\mathrm{C} 28$ & $-4.8(5)$ \\
\hline $\mathrm{C} 4-\mathrm{C} 5-\mathrm{C} 6-\mathrm{C} 10$ & $-47.7(3)$ & $\mathrm{C} 17-\mathrm{C} 18-\mathrm{C} 19-\mathrm{C} 20$ & $2.3(4)$ \\
\hline $\mathrm{C} 6-\mathrm{O} 1-\mathrm{C} 7-\mathrm{C} 8$ & $-69.4(3)$ & $\mathrm{C} 28-\mathrm{C} 18-\mathrm{C} 19-\mathrm{C} 20$ & $-176.1(3)$ \\
\hline
\end{tabular}




\begin{tabular}{|c|c|c|c|}
\hline $\mathrm{C} 6-\mathrm{O} 1-\mathrm{C} 7-\mathrm{C} 25$ & $47.9(3)$ & $\mathrm{C} 18-\mathrm{C} 19-\mathrm{C} 20-\mathrm{C} 21$ & $2.1(5)$ \\
\hline $\mathrm{O} 1-\mathrm{C} 7-\mathrm{C} 8-\mathrm{O} 3$ & $-149.9(3)$ & $\mathrm{C} 19-\mathrm{C} 20-\mathrm{C} 21-\mathrm{C} 22$ & $-3.8(4)$ \\
\hline $\mathrm{C} 25-\mathrm{C} 7-\mathrm{C} 8-\mathrm{O} 3$ & $98.7(3)$ & $\mathrm{C} 1-\mathrm{N} 1-\mathrm{C} 22-\mathrm{C} 21$ & $178.2(3)$ \\
\hline $\mathrm{O} 1-\mathrm{C} 7-\mathrm{C} 8-\mathrm{C} 9$ & $31.6(4)$ & $\mathrm{C} 1-\mathrm{N} 1-\mathrm{C} 22-\mathrm{C} 17$ & $-0.6(3)$ \\
\hline $\mathrm{C} 25-\mathrm{C} 7-\mathrm{C} 8-\mathrm{C} 9$ & $-79.8(3)$ & $\mathrm{C} 20-\mathrm{C} 21-\mathrm{C} 22-\mathrm{N} 1$ & $-177.4(3)$ \\
\hline $\mathrm{O} 3-\mathrm{C} 8-\mathrm{C} 9-\mathrm{C} 10$ & $-174.8(3)$ & $\mathrm{C} 20-\mathrm{C} 21-\mathrm{C} 22-\mathrm{C} 17$ & $1.2(4)$ \\
\hline $\mathrm{C} 7-\mathrm{C} 8-\mathrm{C} 9-\mathrm{C} 10$ & $3.5(4)$ & $\mathrm{C} 18-\mathrm{C} 17-\mathrm{C} 22-\mathrm{N} 1$ & $-178.0(3)$ \\
\hline $\mathrm{C} 8-\mathrm{C} 9-\mathrm{C} 10-\mathrm{C} 11$ & $-170.2(3)$ & $\mathrm{C} 16-\mathrm{C} 17-\mathrm{C} 22-\mathrm{N} 1$ & $0.9(3)$ \\
\hline $\mathrm{C} 8-\mathrm{C} 9-\mathrm{C} 10-\mathrm{C} 6$ & $1.0(4)$ & $\mathrm{C} 18-\mathrm{C} 17-\mathrm{C} 22-\mathrm{C} 21$ & $3.1(4)$ \\
\hline $\mathrm{O} 1-\mathrm{C} 6-\mathrm{C} 10-\mathrm{C} 9$ & $-40.5(4)$ & $\mathrm{C} 16-\mathrm{C} 17-\mathrm{C} 22-\mathrm{C} 21$ & $-178.0(3)$ \\
\hline $\mathrm{O} 2-\mathrm{C} 6-\mathrm{C} 10-\mathrm{C} 9$ & $72.5(3)$ & $\mathrm{C} 6-\mathrm{O} 2-\mathrm{C} 25-\mathrm{C} 26$ & $128.7(3)$ \\
\hline $\mathrm{C} 5-\mathrm{C} 6-\mathrm{C} 10-\mathrm{C} 9$ & $-162.8(3)$ & $\mathrm{C} 6-\mathrm{O} 2-\mathrm{C} 25-\mathrm{C} 27$ & $-108.7(3)$ \\
\hline $\mathrm{O} 1-\mathrm{C} 6-\mathrm{C} 10-\mathrm{C} 11$ & $131.5(3)$ & $\mathrm{C} 6-\mathrm{O} 2-\mathrm{C} 25-\mathrm{C} 7$ & $6.5(3)$ \\
\hline $\mathrm{O} 2-\mathrm{C} 6-\mathrm{C} 10-\mathrm{C} 11$ & $-115.5(3)$ & $\mathrm{O} 1-\mathrm{C} 7-\mathrm{C} 25-\mathrm{O} 2$ & $-33.0(3)$ \\
\hline $\mathrm{C} 5-\mathrm{C} 6-\mathrm{C} 10-\mathrm{C} 11$ & $9.2(4)$ & $\mathrm{C} 8-\mathrm{C} 7-\mathrm{C} 25-\mathrm{O} 2$ & $84.1(3)$ \\
\hline $\mathrm{C} 9-\mathrm{C} 10-\mathrm{C} 11-\mathrm{O} 4$ & $102.7(3)$ & $\mathrm{O} 1-\mathrm{C} 7-\mathrm{C} 25-\mathrm{C} 26$ & $-150.7(3)$ \\
\hline $\mathrm{C} 6-\mathrm{C} 10-\mathrm{C} 11-\mathrm{O} 4$ & $-68.5(3)$ & $\mathrm{C} 8-\mathrm{C} 7-\mathrm{C} 25-\mathrm{C} 26$ & $-33.7(4)$ \\
\hline $\mathrm{C} 9-\mathrm{C} 10-\mathrm{C} 11-\mathrm{C} 12$ & $-15.1(4)$ & $\mathrm{O} 1-\mathrm{C} 7-\mathrm{C} 25-\mathrm{C} 27$ & $81.9(3)$ \\
\hline $\mathrm{C} 6-\mathrm{C} 10-\mathrm{C} 11-\mathrm{C} 12$ & $173.7(2)$ & $\mathrm{C} 8-\mathrm{C} 7-\mathrm{C} 25-\mathrm{C} 27$ & $-161.0(3)$ \\
\hline $\mathrm{C} 9-\mathrm{C} 10-\mathrm{C} 11-\mathrm{C} 3$ & $-143.1(3)$ & $\mathrm{C} 19-\mathrm{C} 18-\mathrm{C} 28-\mathrm{C} 31$ & $-118.9(3)$ \\
\hline $\mathrm{C} 6-\mathrm{C} 10-\mathrm{C} 11-\mathrm{C} 3$ & $45.7(3)$ & $\mathrm{C} 17-\mathrm{C} 18-\mathrm{C} 28-\mathrm{C} 31$ & $62.7(3)$ \\
\hline $\mathrm{C} 24-\mathrm{C} 3-\mathrm{C} 11-\mathrm{O} 4$ & $165.8(2)$ & $\mathrm{C} 19-\mathrm{C} 18-\mathrm{C} 28-\mathrm{C} 29$ & $0.4(4)$ \\
\hline $\mathrm{C} 2-\mathrm{C} 3-\mathrm{C} 11-\mathrm{O} 4$ & $-73.9(3)$ & $\mathrm{C} 17-\mathrm{C} 18-\mathrm{C} 28-\mathrm{C} 29$ & $-178.0(3)$ \\
\hline $\mathrm{C} 4-\mathrm{C} 3-\mathrm{C} 11-\mathrm{O} 4$ & $49.9(3)$ & $\mathrm{C} 19-\mathrm{C} 18-\mathrm{C} 28-\mathrm{C} 30$ & $118.4(3)$ \\
\hline $\mathrm{C} 24-\mathrm{C} 3-\mathrm{C} 11-\mathrm{C} 10$ & $54.8(3)$ & $\mathrm{C} 17-\mathrm{C} 18-\mathrm{C} 28-\mathrm{C} 30$ & $-60.0(3)$ \\
\hline $\mathrm{C} 2-\mathrm{C} 3-\mathrm{C} 11-\mathrm{C} 10$ & $175.1(2)$ & $\mathrm{C} 18-\mathrm{C} 28-\mathrm{C} 31-\mathrm{C} 32$ & $29.3(4)$ \\
\hline $\mathrm{C} 4-\mathrm{C} 3-\mathrm{C} 11-\mathrm{C} 10$ & $-61.1(3)$ & $\mathrm{C} 29-\mathrm{C} 28-\mathrm{C} 31-\mathrm{C} 32$ & $-94.0(4)$ \\
\hline $\mathrm{C} 24-\mathrm{C} 3-\mathrm{C} 11-\mathrm{C} 12$ & $-72.4(3)$ & $\mathrm{C} 30-\mathrm{C} 28-\mathrm{C} 31-\mathrm{C} 32$ & $150.1(3)$ \\
\hline $\mathrm{C} 2-\mathrm{C} 3-\mathrm{C} 11-\mathrm{C} 12$ & $47.9(3)$ & & \\
\hline
\end{tabular}

Hydrogen-bond geometry $\left(A,{ }^{\circ}\right)$

$\mathrm{Cg}$ is the centroid of the $\mathrm{C} 17-\mathrm{C} 22$ ring.

\begin{tabular}{lllll}
\hline$D-\mathrm{H} \cdots A$ & $D-\mathrm{H}$ & $\mathrm{H} \cdots A$ & $D \cdots A$ & $D-\mathrm{H} \cdots A$ \\
\hline $\mathrm{O} 4-\mathrm{H} 4 \cdots \mathrm{O}^{\mathrm{i}}$ & 0.82 & 2.03 & $2.757(3)$ & 148 \\
$\mathrm{~N} 1-\mathrm{H} 1 \cdots C g^{\mathrm{ii}}$ & 0.86 & 2.78 & $3.527(1)$ & 146 \\
\hline
\end{tabular}

Symmetry codes: (i) $-x+2, y-1 / 2,-z+2$; (ii) $-x+1, y-1 / 2,-z+1$. 